\title{
An Integrated Service Model for Improved Automatic Teller Machine Operation in Nigeria Banking System
}

\author{
Oriola Oluwafemi \\ Department of Computer Science, \\ Adekunle Ajasin University, \\ Akungba Akoko, Nigeria.
}

\author{
Aranuwa Felix Ola \\ Department of Computer Science, \\ Adekunle Ajasin University, \\ Akungba Akoko, Nigeria.
}

\begin{abstract}
Nigeria economy until recently depended on cash transactions. By Central Bank of Nigeria (CBN) Act 2007, cashless banking through Automated Teller Machine (ATM) was officially introduced into some segments of Nigeria Banking sector to improve banking operation and bring about fiscal growth of the economy. The cashless programmes launched included ATM, Debit Card and Credit Card transactions. The popularity of transactions through ATM can be ascribed to convenience of use. However, at present in Nigeria, ATM is not satisfying the set objectives in the area of transaction reliability, compliance of process, and transaction response time thereby resulting to ATM fraud and security flaws; delay operations; and erroneous reporting and feedback. To a large extent, these problems can be attributed to defective policy framework and implementation. Therefore, this paper focuses on improving the operation of ATM using the combination of Service Component Architecture (SCA) and Case Based Reasoning (CBR) as a framework for Service Oriented Computing. It describes a new service oriented modeling approach where the SCA is used to facilitate the composition of service within ATM operation while the CBR is used to execute the service functions selected by users. These models are relevant to Commercial Bank and CBN layers of Service Model under the regulation of CBN based on customers' preferences over customers' requirements via the ATM and internet banking interface. The model serves as a framework that could guarantee better operation in ATM banking.
\end{abstract}

\section{General Terms}

Central Bank of Nigeria, ATM Fraud, Nigeria Economy

\section{Keywords}

Automated Teller Machine, Service Oriented Architecture, Service Component Architecture, Case Based Reasoning, Service Execution

\section{INTRODUCTION}

Automated Teller Machines (ATMs) were introduced in the 1970s to reduce the need for bank tellers and to provide convenience for bank customers [1]. Today, ATM is important part of everyday life. According to statistics, there is one Automated teller machine for every 284 American households. Nearly 11 billion transactions are conducted through ATM each year. ATM has become a competitive weapon to a commercial bank for multiple reasons. First, automation replaces labour and improves the traditional labour transaction process. With the introduction of automated equipment, the advantages of automation such as reduction in labour errors and shortened processing time will increase efficiency of banks and improve service quality [2]. According to Peter and Sylvia (2008)[3], an ATM combines a computer terminal, record keeping system, and cash vault in one unit, permitting customers to enter a financial firm's bookkeeping system with either a plastic card containing a Personal Identification Number (PIN) or by punching a special code number into a computer terminal linked to the financial firm's computerized records 24 hours a day. Once access is gained into the system, cash withdrawals may be made up to pre-specified limits and balance inquiries, and bill paying may take place. Mishkin (1998)[4] states that the wonders of modern computer technology have also enabled banks to lower the cost of bank transactions by having the customer interact with an electronic banking facility rather than with a human being. In Nigeria, a mechanical cash dispenser, arguably an ATM was introduced in 1986 by the defunct Societe General Bank. In October 2003 InterSwitch ATM system took off. ATM services have a history that is less than fifteen years in Nigeria. At first, they were operated as elitist services designed for those desirous of exclusive service. Cards were rare and the process for obtaining them tortuous. Presently, the use of ATM cards has been widely promoted. Banks no longer appear to want personal contact with their customers. Some banks have resorted to penalizing the customer as it were, for not possessing an ATM card, by debiting the account of such a customer for withdrawing below a certain amount across the counter. Agboola (2006) [5] reported that although only a bank had an ATM in 1998, by 2004 , fourteen of them had acquired the technology.

The adoption of ICT in banks has produced largely positive outcomes such as improved customer services, more accurate records, ensuring convenience in business time, prompt and fair attention, and faster services. In addition, the banks' image has improved, creating a more competent market. Work has also been made easier, and more interesting, the competitive edge of banks, relationship with customers, and the solution of basic operational and planning problems has been improved. The number of ATM transactions through the Interswitch network has considerably increased from, $1,065,972$ in 2004 , to $14,448,615$ between January 2005 to March 2006. This is a rise of 92.6 percent with respect to the previous years. More than 800 ATMs have been deployed on the network, while about 2 million cards have been issued by 23 banks as at March 2006 [6]

ATMs are used by all modern commercial banks and are found in cities around the world. Modern ATMs have addressed many human interaction concerns; however its operation still suffers from a number of challenges. The following challenges are critical in ATM improvements. 
Security: The issue of security in this environment cannot be over emphasized. ATM fraud and security flaws need to be prevented. Its process is expected to focus more on customer/transactions security through multi-level process of password and biometric based security applications [7].

Functionality: Traditional ATMs presently implement most basic daily banking functions, such as deposit, withdrawals and balance checking. The performance limitations of computing and networking currently being experienced in the operation should be improved upon to enable the system to handle as many traditional teller operations as possible effectively and efficiently. Hence the next-generation ATM should support the following features:

- Efficiency: ATMs is expected to be both easy to use and fast. The more time a user spends at an ATM, the more inconvenienced the user feels. This also increases the waiting time for other users. Clearly, this is an important area been considered in the framework design in this work.

- Accessibility: ATMs are physical machines to serve generality of users; hence their design must take accessibility concerns into account. Some good accessibility features should includes Braille support for the blind. Modern ATM design should be convenient for all users.

- Other challenges pertaining to execution of service by service providers who can either be Central Bank or Commercial Banks include late response to request, reliability and correctness issues, cash withdrawal denial by ATM leaving customers stranded for days, inadequacy and wastage of resources.

Thus, this study focuses on improving deployment, maintenance, security and regulatory monitoring of ATM and its operations in Nigeria Banking System.

\section{BACKGROUND OF THE STUDY 2.1 Roles of Service Providers}

According to CBN Act (2007) [2], there are roles that pertain to ATM operation by Commercial Banks and CBN. These roles are discussed below:

\subsubsection{Commercial Bank Service}

i. Deployment

A bank or independent organization that deploys an ATM for the use of the public shall ensure that:

(a). The ATM downtime (due to technical fault) is not more than seventy-two (72) hours consecutively;

(b). The helpdesk contacts should be adequately displayed at the ATM terminals. At the minimum, a telephone line should be dedicated for fault reporting and such telephone facility shall be functional and manned at all times.

(c). All ATM surcharges are fully disclosed to customers;

(d). The ATMs should issue receipts, where requested by a customer, for all transactions, except for balance enquiry, stating at a minimum the amount withdrawn, the surcharges, date and time of the transaction;

(e). Receipt prints and screen display should be legible. (f). The dispensing deposit and recycling component of the machine is in proper working condition;

(g). The dispensing component holds out the notes for the collection of the user for a minimum of twenty (20) seconds;

(h). There is appropriate monitoring mechanism to determine failure to dispense cash;

(i). There is online monitoring mechanism to determine ATM vault cash levels

(j). ATM vault replenishment is carried out as often as possible to avoid cash-out.

(k). ATMs are not stocked with unfit notes;

(l). Availability of cash in the ATMs at all time. The funding and operation of the ATM deployed by non-bank institutions should be the sole responsibility of the bank or institutions that entered into agreement with them for cash provisioning. In this regard, the Service Level Agreement (SLA) should specify the responsibilities of each of the parties.

(m). Change of PIN is provided to customers free of charge throughout the entire value chain.

(n). Acquirers monitor suspicious transactions and report statistics to CBN based on the agreed format and timeframe

(o). Back-up power (inverter) is made available at all ATM locations in such a way that the machine would not cease operation while in the middle of a transaction.

(p). Waste disposal basket is provided at all ATM locations

(q). A register of all their ATMs in Nigeria with location, identification, serial number of the machines, etc is maintained.

(r). Provision is made for extending the time needed to perform a specific step by presenting a question, such as, "Do you need more time?"

(s). Information sufficient to construct a usable card is not displayed on the screen or printed on a transaction record. This will guard against the possibility that such information may become accessible to another person should the cardholder leave the ATM while a transaction is displayed, or abandon a printed transaction record.

(t). Precautions are taken to minimize the possibility of a card being left by a message or voice alerting the customer to take his card.

(u). Cash out first before card is out of the ATM is adopted to minimize the possibility of customers leaving cash uncollected at ATM.

(v). ATM acquirers that disable cash-retract shall display such notice at the ATM or on the screen.

\section{ii. ATM Maintenance}

A bank or independent organization that deploys an ATM for the use of the public shall ensure that:

(a). Notice is displayed at the ATM for planned maintenance period and disruption to service due to maintenance for public;

(b). An ATM maintenance register or log is kept properly

(c). All ATMs and cash in the machines are insured.

(d). They physically inspect their ATMs at least fortnightly.

\section{iii. ATM Security}

(a). Every ATM shall have functioning cameras which shall view and record all persons using the machines and every activity at the ATM including but not limited to: card insertion, PIN entry, transaction selection, cash withdrawal, card taking, etc. However, such cameras should not be able to record the key strokes of customers using the ATM. 
(b). Where a surveillance camera is used, it should be kept secretly to avoid thieves removing or damaging or compromising it.

(c). Networks used for transmission of ATM transactions must be demonstrated to have data confidentiality and integrity.

(d). All ATMs must be located in such a manner that guarantees safety and security of users and confidentiality of their transactions.

(e). ATMs should not be placed outside buildings unless such ATM is bolted to the floor and surrounded by structures to prevent removal.

(f). Additional precaution must be taken to ensure that any network connectivity from the ATM to the bank or switch is protected to prevent the connection of other devices to the network point.

(g). Where the user of an ATM blocks his image for camera capture, the ATM shall be capable of aborting the transaction.

\section{iv. Irregularities Resolution}

In the event of irregularities in the account of an ATM customer arising from the use of card on ATM, the following shall apply:

(a). All cardholders' complaints should be treated within a maximum of 72 hours from the date of receipt the complaints.

\section{v. Liability Shift}

(a). Where a non EMV card is used on a non EMV Terminal and a fraud occurs, liability is on either the Card Issuer or the Card Holder. Proof has to be established on which party compromised card details.

(b). Where a non EMV card is used on an EMV Terminal and fraud occurs, liability is on the Card Issuer

(c). Where an EMV card is used on a non EMV Terminal and fraud occurs, liability is on the Acquirer

(d). Where an EMV card is used on an EMV Terminal and fraud occurs, liability is on the Card Holder or the Issuer. However, the onus is on the cardholder to prove that their PIN had not been disclosed to a third party willingly or negligently.

(e). Where a hybrid card is used on a non EMV Terminal and fraud occurs, liability is on the Acquirer.

(f). Where a hybrid card is used on an EMV Terminal and card treated as magnetic stripe for authorization and fraud occurs, liability is on the Card Issuer.

(g). Where a hybrid card is used on an EMV Terminal and card treated as EMV for authorization and fraud occurs, liability is on the Card Holder or the Issuer. However, the onus is on the cardholder to prove that his/her PIN had not been disclosed to a third party willingly or negligently.

\subsubsection{Central Bank Service}

i. Regulatory Monitoring

(a). Any institution which operates an automated teller machine shall file an updated list of such ATMs, including the detail location of their addresses with Banking \& Payments System Department of the Central Bank of Nigeria for compliance monitoring.

(b). The CBN shall conduct onsite snap checking of ATMs with a view to ensuring compliance with cash and service availability at the ATMs. (c). Acquirers shall report volume and value of transactions on monthly basis to the Director, Banking \& Payments System Department, CBN.

ii. Penalties

Sanctions, in the form of monetary penalties / or suspension of the acquiring/processing service (s) or both, would be imposed on erring institutions for failure to comply with any of the provisions of the ATM standards and guidelines or any other relevant guidelines issued by the CBN from time to time.

\subsection{Service Oriented Architecture}

Service-oriented architecture (SOA) is an evolution of distributed computing based on the request/reply design paradigm for synchronous and asynchronous applications [8]. It is described as an architecture design pattern based on distinct pieces to provide application functionality as services to other applications. Service-Oriented Architecture (SOA) offers way to view sensors, objects, participants as services. It can provide an effective approach to improve their communication and coordination, so that the human participants can effectively interact with the system and offer their opinions and preferences through a coherent technology. SOA could be a potential approach to maximize users' satisfaction degree, and minimize the cost of software design, implementation, application installation, and system maintenance. Most service discovery and recommending systems recommend a list of services according to the functional and/or non-functional requirements given by service consumers. Thus, the service consumer can choose the best one. This kind of cases is based on an assumption that the cardinality between an instance of service and a service consumer is built upon one to one relationship. SOA offers the following advantages: decentralized middleware and loose coupling. The loose coupling is associated with capability such as discovery by abstract descriptions, evolvable systems independent of implementation/platform, interchangeable by interface definition, autonomous services, and reusable services in new contexts and inter-organizational [9].

\subsection{Service Component Architecture}

According to OASA (2009)[10], Service Component Architecture (SCA) is a set of specifications which describe a model for building applications and systems by composing and deploying new and existing service components. SCA builds upon an open standard such as Web services and complements to the existing approaches in service implementation. It is based on the idea that business function is supported by a series of services, which can be assembled together to create solutions and to serve a particular business need. The composite applications can contain both new services and business functions from existing systems and applications [11], [12].

SCA specifies how those components can be combined into larger structures called composites. A composite is a logical construct, and its components can run in a single process on a single computer or be distributed across multiple processes on multiple computers. Furthermore, SCA is aimed to encompass a wide range of technologies to support service component accessibility and web service connectivity [13]. For components, this includes not only different programming languages, but also framework and environments commonly 
used with those languages. The SCA specifications are divided into a number of documents such as assembly model, policy framework, transaction policy, Web Service binding, JMS binding, and each document is responsible to one aspect of SCA. SCA assembly model deals with the aggregation of components and their linkages. The Assembly Model is independent of implementation language which deals with the aggregation of components and the linking of components through wiring using composites. In SCA, a composite is a collection, or assembly, of components or services. A composite considered a "service" when it's wrapped for external consumption. Furthermore, a composite can contain properties and reference to other components or services, while a component is not necessarily intended to be exposed for external consumption. A service in SCA is a selfcontained unit of functionality. It can be classified into atomic or composite services. The benefits of building software based on the reusable building blocks of components and composites have been espoused for several decades. Moreover, SCA does not only extend and complement prior approaches to implementing services, but also provide a programming model for building applications and systems based on a SOA.

\subsection{Case Based Reasoning}

Case-based reasoning is a problem solving paradigm that in many respects is fundamentally different from other major AI approaches. Instead of relying solely on general knowledge of a problem domain, or making associations along generalized relationships between problem descriptors and conclusions, CBR is able to utilize the specific knowledge of previous experiences for concrete problem situations or cases. A new problem is solved by finding a similar past case, and reusing it in the new problem situation [14]. A second important difference is that CBR also is an approach to incremental, sustained learning, since a new experience is retained each time a problem has been solved, making it immediately available for future problems. In CBR terminology, a case usually denotes a problem situation. A previously experienced situation, which has been captured and learned in a way that it can be reused in the solving of future problems, is referred to as a past case, previous case, stored case, or retained case. Correspondingly, a new case or unsolved case is the description of a new problem to be solved. Case-based reasoning is - in effect - a cyclic and integrated process of solving a problem, learning from this experience, to solving a new problem, etc.

Case-based reasoning favours learning from experience, since it is usually easier to learn by retaining a concrete problem solving experience than to generalize from it. Still, effective learning in CBR requires a well worked out set of methods in order to extract relevant knowledge from the experience, integrate a case into an existing knowledge structure, and index the case for later matching with similar cases.

The descriptive framework of CBR is contained in CBR cycle as presented in Figure 1.

At the highest level of generality, a general CBR cycle may be described by the following four Processes:

i. RETRIEVE the most similar case or cases

ii. REUSE map the solution from the previous case to the target problem, use the information and knowledge in that case to solve the problem

iii. REVISE the proposed solution iv. RETAIN the parts of this experience likely to be useful for future problem solving

A new problem is solved by retrieving one or more previously experienced cases, reusing the case in one way or another. It revise the solution based on reusing a previous case, and retaining the new experience by incorporating it into the existing knowledge-base (case base). The four processes each involve a number of more specific steps.

According to Aamodt and Plaza (1994), an initial description of a problem defines a new case. This new case is used to RETRIEVE a case from the collection of previous cases. The retrieved case is combined with the new case - through REUSE - into a solved case, i.e. a proposed solution to the initial problem. Through the REVISE process this solution is tested for success, e.g. by being applied to the real world environment or evaluated by a teacher, and repaired if failed. During RETAIN, useful experience is retained for future reuse, and the case base is updated by a new learned case, or by modification of some existing cases.

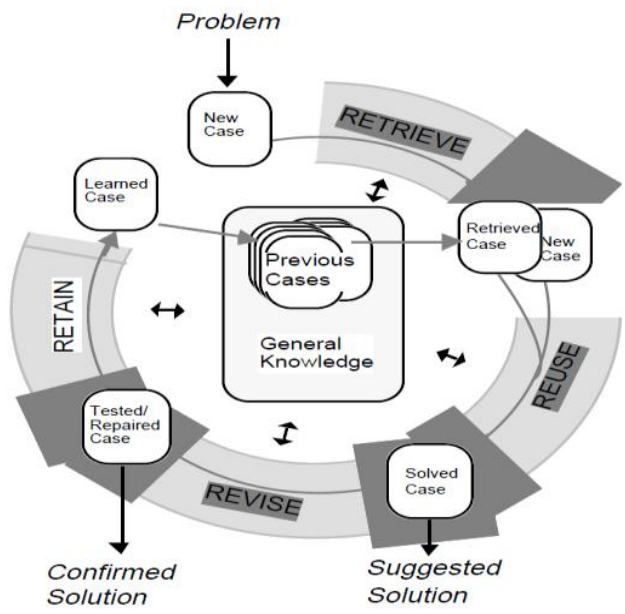

Figure 1: The CBR Cycle [14]

\subsection{Related Works}

Prodem FFP in Bolivia, Banco Ademi in the Dominican Republic, and MEB Kosovo each introduced ATMs in to meet the needs of their unique client populations, communications infrastructures, and costs of participating in ATM networks as presented in Whelan (2009)[15]. They used voice instructions in three languages to assist illiterate or semi-literate users. They offer the primary benefit of convenience for customers and increased deposit mobilization. Customers used the ATMs for many transactions that previously required staff attention making it possible for banks to handle more personalized customer interactions, rather than routine deposit-taking and balance- checking.

Many researches focus on facilitating service-oriented system development by proposing different service discovery protocols to increase accuracy in service discovery and selection [16], [17]. Also, there is Universal Description; Discovery and Integration (UDDI) specification [18] which is one of the most widely used protocols for publishing service that enables service specification to be advertised in a service registry. 
The roots of case-based reasoning in $\mathrm{AI}$ is found in the works of Schank (1982)[19] on dynamic memory and the central role that a reminding of earlier situations (episodes, cases) and situation patterns (scripts, MOPs) has in problem solving and learning. It was used in CYRUS system, developed by Janet Kolodner at Yale University (Schank's group) as reported in Kolodner (1983) [20].

Other trails into the CBR field have come from the study of analogical reasoning Gentner (1983)[21], and theories of concept formation, problem solving and experiential learning within philosophy and psychology [11], [22]. For example, Wittgenstein (1953) [23] observed that 'natural concepts', i.e. concepts that are part of the natural world - such as bird, orange, chair, car, etc. - are polymorphic. That is, their instances may be categorized in a variety of ways, and it is not possible to come up with a useful classical definition, in terms of a set of necessary and sufficient features, for such concepts. An answer to this problem is to represent a concept extensionally, defined by its set of instances - or cases.

\section{ATM OPERATION MODELS}

\subsection{ATM Service Requirements}

The following functional requirements must be met by ATM service providers in accordance with ATM Banking Operational Standards.

- Money Transfer: transfer money from one bank account to another person's bank account.

- Bill Payment: ATM could ensure automatic payment of bills.

- Efficiency: ATMs must be easy to use, fast and efficient. This is an important issue to consider when designing an ATM system.

- Security of Transaction: ATM should serve as a reliable and confidential medium for users.

- Dispense of Money: ATM should be able to dispense accurately the user specified denomination as part of ways of satisfying user need.

- Reliable electrical power and communications infrastructure.

- Affordable dial-up or leased line rates for the ATM to send and receive data.

- A central database where client data must be stored for balance verification.

- Reliable after-sales servicing and support from the vendor or third-party.

- Solid operational procedures and resources to distribute cards and control PINs.

- Supply of correctly denominated currency Systems to securely transfer cash to ATMs.

- Adaptations for effective usage, such as building-in verbal instructions to guide illiterate users.

Other requirements that could affect ATM operation include:

- Issue of payment vouchers, travelers cheques, circular notes etc.

- Establishment of standing guarantee on behalf of ATM customers.

- Collection and supply of business information;

- Issuing of demand drafts and pay orders.,

\subsection{Proposed ATM Service Model \\ 3.2.1 User Service Model}

In operation of ATM, the users that are involved can be classified as Service Consumers and Service Providers. The Service Consumers request services from the Service Providers who ensures proper delivery of the services. The consumers are Commercial Banks' customers that perform transactions via ATM. The Service Providers include Commercial Banks at a lower level and Central Bank of Nigeria at a higher level. In Nigeria, there are CBN branch offices in states that are responsible to the CBN head office in the Federal Capital Territory. The CBN branches are saddled with the responsibility of ensuring that the banks in their states adhere to standard practices while the CBN head office formulates policy framework and oversees the affairs of the CBN branch offices. These users and the corresponding ATM operational components are presented in Figure 4.

At the customer level of service layer, user specifies their requirements using requirement specification language such as mark up languages (html, xml, etc). Using user based modeling tool such as fuzzy linguistic function and controller, customer requirements preference are selected by the commercial bank. At the commercial bank layer, the selected service is broken down into series of atomic composite components which are executed using case based reasoner that is integrated into Service Execution Server at CBN branch offices. At another level of CBN layer, service controller is used to set up policies and regulate the process.

In Figure 5, the architecture of the proposed integrated service model for ATM operation in Nigeria banking environment is presented. The functional steps in the operational framework of the proposed integrated Service Model are presented as follows.

i. Service Collection: Since customers need to express their preferences over their required services, there is a GUI for them to input their opinions. A collection service would gather these inputs and send them to service preference engine.

ii. Service Preference: It consists of fuzzy logic controller that make use of service registry, membership function and inference system in selecting preference services. iii. Service Component Architecture: This component is responsible for breaking and mapping service into component functions and deploying the service to Service Execution Engine. In Figure 5 for instance, the selected service serves as the parent component which is broken into functions. A function that can further be broken into other functions is known as composite components while the functions that cannot be further broken are known as atomic functions represented as leaf or child component (Component A-Component F). Atomic functions are adopted in this framework.

iv. Service Execution Engine: It consists of CBR system and Service Execution Server. The server controls selection of services for execution, implementation of service, evaluation of service and maintenance of service. The execution of these services is actualized using CBR system with operational steps such as retrieve, reuse, revise and retain.

v. Service Registry: The aim of service registry is to store service profiles based on the functionalities provided by the ATM operation. 
vi. Service Controller: This component serves two purposes which include policy regulation and surveillance control. The latter deals with monitoring and supervision of the activities of the $\mathrm{CBN}$ branch offices online while the

ATM User

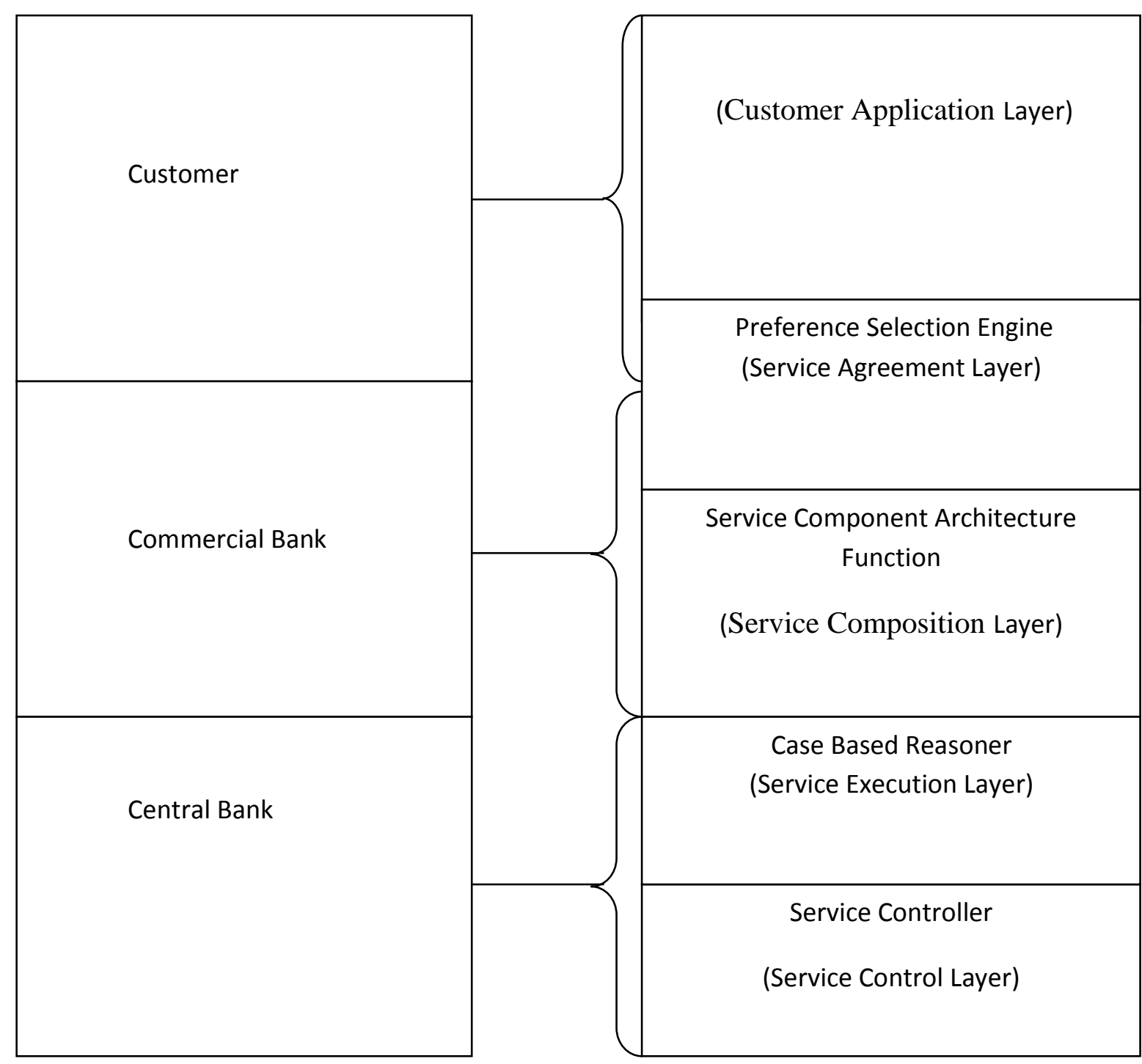

Figure 4: Operational Service Components and Users in ATM Banking former ensures prompt policy set up and regulation compliance.

\section{Operational Components}

\section{(Service Agreement Layer)}

Case Based Reasoner

(Service Execution Layer)

Service Controller

(Service Control Layer) 


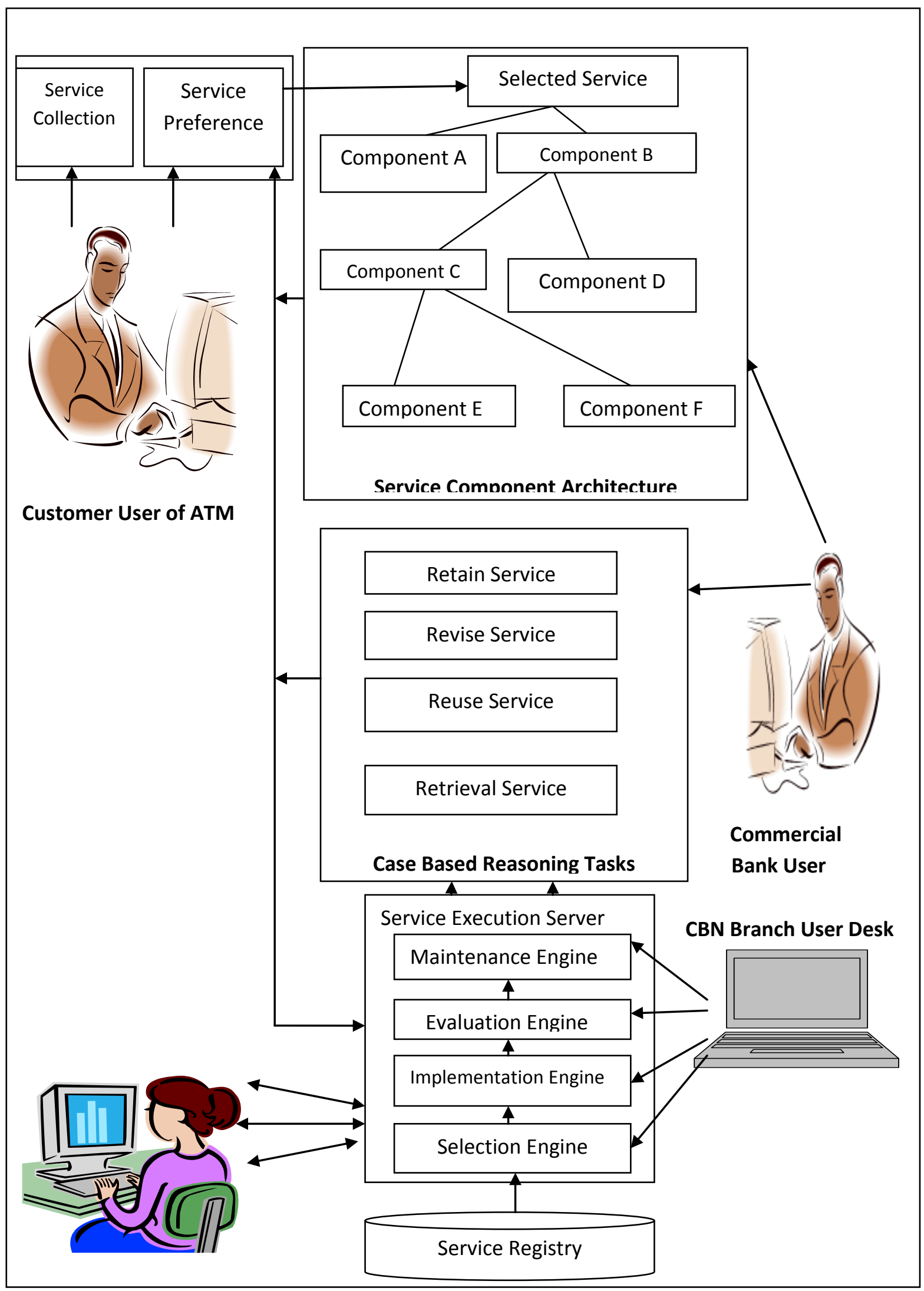

Figure 5: Architecture of the Integrated Service Oriented Framework Showing Interaction among Functional Components 


\section{CONCLUSION}

Although, ATM has potentials of ensuring efficient banking services and improved fiscal growth of the economy. However, it suffers from insecure and fraudulent transaction, inefficient and disrupted services, poor recording and reporting and ineffective control which could be alleviated through institutionalization of proper policy framework and implementation.

This work adopts Service Oriented Computing paradigm that integrate Service Component Architecture and Case Based Reasoning for service selection, implementation, evaluation and maintenance as framework for ATM operational process lifecycle. The model consists of four layers of Components such as Customer Application Layer - Requirement Specification Language, Service Agreement LayerPreference Selection Engine, Service Composition LayerService Component Architecture, Service Execution LayerCase Based Reasoning and Control Layer-Service Controller. The service execution engine or controller can be installed in distributed manner at all CBN offices in states nationwide for timely execution and monitored by a centrally installed surveillance at the $\mathrm{CBN}$ head office for proper monitoring.

The service oriented model serves as framework for ensuring best practices in ATM operation in Nigeria Banking System. Moreover, the model could also be adapted to some domains in the banking system such as internet banking, and the traditional banking operation. In future, works will be carried out on customer requirements preference model. The application and evaluation of the model in real life scenario will also be carried out.

\section{REFERENCES}

[1] http://www.oppapers.com/essays/Automated-TellerMachine-Atm-Nigeria-Present/164303 Accessed April 3, 2009.

[2] CBN Act.2007. Standards and Guidelines on Automated Teller Machine (ATM) Operations in Nigeria.

[3] Peter, S.R. and Sylvia, C.H. 2008. Bank Management and Financial Services. New York: McGraw Hill/Irwin [4] Mishkin, F.S.1998. The Economics of Money, Banking and Financial Markets. U.S.A: Jeffrey Sylvester/FPG International Corporation.

[5] Agboola, A. 2006. Information and communication technology (ICT) in banking operations in Nigeria: an evaluation of recent experiences, Retrieved from http://unpan1.un.org/intradoc/groups/public/documents/ AAPAM/UNPANO 26533. pdf, on Nov. 15, 2011.

[6] CBN, 2011.: Further Clarifications on Cash-less Lagos Project, $($ Central Bank of Nigeria, 2006-2011. All rights reserved.

[7] Aranuwa F.O and Ogunniye G.B (2012). Enhanced Biometric Authentication System for Efficient and Reliable e-Payment System in Nigeria. International Journal of Applied Information Systems (IJAIS) - ISSN : 2249-0868 Foundation of Computer Science FCS, New York, USA Volume 4- No.2, September 2012 www.ijais.org
[8] Raghu R. Kodali (2005) What is service-oriented architecture? An introduction to SOA. JavaWorld, June 13, 2005.

[9] Cardoso, Jorge; Sheth, Amit P. (2006). Semantic Web Services, Processes and Applications. SEMANTIC WEB AND BEYOND: Computing for Human Experience. Foreword by Frank Leymann. Springer. xxi. ISBN $978-$ $\underline{0-387-30239-3}$.

[10] OASA, a set of Final V1.0 Specifications, Open SOA (OSOA), March 2007.

[11] Chen, W., Nguyen S. T., Coops, R., Bambang-Oetomo, S. and Feijs, L. M. G. 2009. Wireless transmission design for health monitoring at neonatal intensive care units. 2nd international symposium on applied sciences in biomedical and communication technologies (ISABEL 2009), Bratislava, Slovak Republic, Nov. 2009.

[12] Zou, Z., Duan, Z. 2006. Building Business Processes or Assembling Service Components: Reuse Services with BPEL4WS and SCA, Proceedings of the European Conference on Web Services (ECOWS'06), Zurich, Switzerland. Washington, DC: IEEE Computer Society, pp138-147, Dec 4-6, 2006.

[13] Edwards, M. (2011) "Service Component Architecture". OASIS. Retrieved 7 April 2011.

[14] Aamodt, A., \& Plaza, E. (1994). Case-based reasoning: Foundational issues, methodological variations, and system approaches. Artificial Intelligence Communications, 7(1), 39-59. HTML

[15] Whelan, S. 2009. Automated Teller Machines. http://www.cgap.org/gm/document-1.9.2757/IT_atm.pdf Accessed April 3, 2012.

[16] Jini Technology Core Platform Specification, v. 2.0, Sun Microsystems, June 2003.

[17] Service Location Protocol, v. 2, IETF RFC 2608, June 1999.

[18] Bellwood, T., Clement, L., Riegen, C.V. 2004. UDDI Version 3.0.2, UDDI Spec Technical Committee Specifcation.

[19] Schank R. 1982. Dynamic memory; a theory of reminding and learning in computers and people. Cambridge University Press. 1982.

[20] Kolodner, J. Maintaining organization in a dynamic long-term memory. Cognitive Science, Vol.7, pp.243280. 1983.

[21] Gentner, D. 1983. Structure mapping - a theoretical framework for analogy. Cognitive Science, Vol.7. pp.155-170.

[22] Tulving, E. 1977. Episodic and semantic memory. In E. Tulving and W. Donaldson: Organization of memory, Academic Press, 1972. pp. 381-403.

[23] Wittgenstein, L. 1953. Philosophical investigations. Blackwell, pp. 31-34. 